\title{
Using biomarkers to improve the diagnostic value of ductal fluid for early breast cancer detection B Haddad
}

Address: Georgetown University, Lombardi Comprehensive Cancer Center, Washington, DC, USA

Email: B Haddad - Haddadb1@georgetown.edu

from 6th International Symposium on the Intraductal Approach to Breast Cancer

Santa Monica, CA, USA. |9-2| February 2009

Published: 24 July 2009

BMC Proceedings 2009, 3(Suppl 5):S2I doi:I0.1 I86/I753-656I-3-S5-S2 I

This abstract is available from: http://www.biomedcentral.com/I753-656I/3/S5/S2 I

(c) 2009 Haddad; licensee BioMed Central Ltd.

The optimal method for early detection of breast cancer in premenopausal women is not known. To date, mammographic screening and physical exam are the best available approaches for early detection in the general population. However, these methods may not be sufficient in young women. Therefore, there is an urgent need for new approaches that can augment mammographic screening and physical exam, particularly for women at high risk for developing breast cancer, such as carriers of BRCA1/2 mutations, who are known to face markedly elevated lifetime risks for cancer. Therefore, women at high risk constitute the population that will benefit most from new breast screening technologies and early detection strategies.

Mammary epithelial cells destined to progress to cancer may have accumulated a number of premalignant genetic changes, prior to the onset of cancer. Therefore, it may be possible to improve upon cytologic assessment of these cells by simultaneously screening for genomic aberrations. This provides a realistic opportunity to improve the detection of premalignant breast changes prior to the onset of cancer. There have been several reports showing the feasibility of studying individual, or a combination of two biomarkers, to assess the ductal fluid. However, no studies have been performed where a panel of multiple biomarkers were evaluated simultaneously, using the same fluid sample. This is particularly significant since it is not clear which biomarker(s) will be most useful for early detection of breast cancer, making a panel of markers more desirable than an individual one. We believe that we can significantly improve the diagnostic value of ductal fluid by maximizing the information one can derive from each specimen. 